\section{Discussion}

The underlying causes of the variation in waiting times observed here are not explained by the audit data, and we do not suggest that there are "across the board" implications for clinical outcome in the waiting times reported. Clearly, these are clinical issues and would vary with specific types of cancer. However, the focus on reducing unnecessary delays in cancer treatment stems from the belief that the earlier disease is detected, the more quickly multidisciplinary care can be instigated and the better the outcome. Irrespective of the specific waiting times reported here, the key contribution of this study is in establishing baseline data which can be used to set targets for improvement and, crucially, to monitor such attempts.
We thank Dr Stephen Green for his advice on the development of the guidance notes used by the trusts in completing the survey instrument and the many trust staff who gave their time in the collection of the data.

Contributors: PS coordinated the study and helped design the survey instrument and write the report. FB analysed the data and helped write the report. DK helped initiate the research, interpret the data, and write the report.

Funding: The study was funded by the Department of Health (HSC1998/999)

Competing interests: None declared.

1 Coleman MP, Babb P, Damiecki P, Grosclaude P, Honjo S, Jones J, et al. Cancer survival trends in England and Wales 1971-1995: deprivation and NHS region. London: Stationery Office, 1999.

2 Berrino F, Capocaccia R, Estéve J, Gatta G, Hakulinen T, Micheli A, et al Survival of cancer patients in Europe: the EUROCARE study II. Lyons: IARC 1999. (IARC Scientific Publication No 151.)

3 National Cancer Institute. SEER Stat-cancer incidence public use database 1973-95. Release 1.1. Bethesda MD: NCI, 1998.

(Accepted 2 March 2000)

\title{
Relation between infants' birth weight and mothers' mortality: prospective observational study
}

\author{
George Davey Smith, Seeromanie Harding, Michael Rosato
}

Several studies have shown inverse associations between birth weight and incidence of cardiovascular disease in adulthood, ${ }^{2}$ suggesting that development in early life may influence the risk of disease many years later. The existence of intergenerational influences on birth weight, illustrated by correlations between the birth weight of parents and the birth weight of their offspring, suggests that birth weight of offspring should be associated with the risk of mortality from cardiovascular disease among parents. So far only one small study has investigated this possibility. ${ }^{3}$ In that study each kilogram decrease in birth weight of the infant was associated with a doubling in the risk of parental mortality from cardiovascular disease. We have replicated this potentially important finding in the Office for National Statistics' longitudinal study, a follow up of $1 \%$ of the population of England and Wales from the 1971 census. $^{4}$

\section{Methods and results}

In the longitudinal study, information from birth registrations of infants during 1976-97 is linked to data from the census and death registration (1976-97) for study members. Birth weight was collected from 1976 onwards, but during the 1970 s the data were not complete. The first recorded birth weight of infants to 44813 women aged 15-45 years at birth registration was used as the exposure measure. Mean birth weight was $3210 \mathrm{~g}$ (SD $566 \mathrm{~g}$ ). The average birth weight changed little over the study period ( $4.6 \mathrm{~g}$ increase a year, $95 \%$ confidence intervals $3.6 \mathrm{~g}$ to $5.6 \mathrm{~g}$ ). The difference between average male birth weight and average female birth weight $(99 \mathrm{~g})$ was subtracted from each male birth weight. Socioeconomic position (housing tenure and car access) of mothers was taken from the 1971 census.
Mortality was analysed by using Cox's proportional hazards model in relation to birth weight, with adjustment for socioeconomic position and whether the birth occurred inside or outside marriage (with additional adjustment for sole registration or joint registration if outside marriage).

The table shows a substantial association between infants' birth weight and mothers' mortality from all causes and from cardiovascular disease. The relative risk of maternal cardiovascular mortality associated with a $1 \mathrm{~kg}$ lower birth weight in offspring was closely similar to that seen in the previous study of this issue (2.00 (1.18 to 3.33$\left.)^{3}\right)$; the corresponding associated risks for the other two major causes of death (153 deaths from cancer (1.33; 1.03 to 1.72$)$ and 38 accidental or violent deaths (1.06; 0.61 to 1.85$)$ ) were considerably smaller than those for cardiovascular mortality.

Department of Social Medicine, University of Bristol, Canynge Hall, Bristol BS8 2PR

George Davey Smith professor of clinical epidemiology

Office for National Statistics, London SW1V 2QQ

Seeromanie

Harding senior research officer Michael Rosato senior research officer

Correspondence to: G Davey Smith zetkin@bristol.ac.uk

BMJ 2000;320:839-40

\section{Comment}

We found a strong inverse relation between infants' birth weights and mothers' mortality from cardio-

Infants' birth weight and mothers' mortality, 1976-97, according to data from Office for National Statistics' longitudinal study (England and Wales). Values are hazard ratios (95\% confidence interval) unless stated otherwise

\begin{tabular}{lccc} 
& No of & \multicolumn{2}{c}{ Deaths of mothers } \\
\cline { 3 - 4 } Infants' birth weight $(\mathbf{g})$ & $\mathbf{b i r t h s}$ & $\begin{array}{c}\text { From all causes } \\
(\mathbf{n = 2 9 3 )}\end{array}$ & $\begin{array}{c}\text { From cardiovascular disease } \\
(\mathbf{n}=\mathbf{4 1})\end{array}$ \\
\hline$<2500$ & 3891 & $3.06(2.15$ to 4.35$)$ & $7.05(2.64$ to 18.77$)$ \\
\hline $2500-3499$ & 27360 & $1.27(0.96$ to 1.69$)$ & $1.96(0.80$ to 4.82$)$ \\
\hline$\geqslant 3500$ & 13562 & 1.00 & 1.00 \\
\hline Per $1000 \mathrm{~g}$ & & $1.63(1.37$ to 1.94$)$ & $2.25(1.48$ to 3.41$)$ \\
\hline Per $1000 \mathrm{~g} \mathrm{(adjusted)}{ }^{*}$ & & $1.61(1.35$ to 1.92$)$ & $2.22(1.46$ to 3.38$)$ \\
\hline
\end{tabular}

*Adjusted for socioeconomic position (housing tenure and car access) at the start of the study in 1971 and marital status (sole or joint registration if unmarried) at birth registration.

Crown copyright reserved. 
vascular disease. Three basic processes could generate this association. Firstly, poor social circumstances could lead to both lower birth weight and higher mortality risk. Secondly, maternal health, nutritional, and behavioural profiles could influence both birth weight and cardiovascular mortality. Thirdly, intergenerational factors-such as genomic and epigenetic processes that lead to a positive correlation between the birth weights of mothers and their offspring-could influence cardiovascular risk.

Adjustment for socioeconomic position and marital status had little influence on the findings in either the current study or the previous investigation of this issue, ${ }^{3}$ rendering a simple explanation in terms of socioeconomic confounding unlikely. In the current study we had no data on health status, but in the earlier study adjustment for a wide range of measures of health and health related behaviours reduced only slightly the association between infants' birth weight and mothers' cardiovascular mortality. ${ }^{3}$ The magnitude of the association in the current study is too great to be generated plausibly by the known associations between birth weight and maternal smoking, alcohol drinking, or anthropometry. ${ }^{5}$
The marked similarity between the current findings and those from the previous study - which related to an earlier generation of women living in widely different circumstances-suggests that an important influence is being uncovered by our analyses. Possible intergenerational influences on birth weight and cardiovascular risk therefore merit further investigation.

Contributors: GDS formulated the hypothesis regarding infants' birth weight and mothers' mortality. All authors discussed and specified the analyses, which were carried out by SH and MR. The first draft of the paper was written by GDS, with SH and MR contributing to the final draft. GDS is the guarantor of the paper. Funding: None.

Competing interests: None declared.

1 Barker DJP. Mothers, babies and disease in later life. London: BMJ Publishing, 1994.

2 Frankel SJ, Elwood P, Sweetnam P, Yarnell J, Davey Smith G. Birthweight, adult risk factors and incident coronary heart disease: the Caerphilly study, Public Health 1996;110:139-43.

3 Davey Smith G, Hart C, Ferrell C, Upton M, Hole D, Hawthorne V, et al. Birth weight of offspring and mortality in the Renfrew and Paisley study: prospective observational study. BMJ 1997;315:1189-93.

4 Goldblatt PO, ed. Longitudinal study: mortality and social organisation. London: HMSO, 1990.

5 Kramer M. Determinants of low birthweight: methodological assessment and meta-analysis. Bull WHO 1987;65:663-737.

(Accepted 7 December 1999)

\title{
Cohort study of birthweight, mortality, and disability
}

\author{
Chris Power, Leah Li
}

Department of Epidemiology and Public Health, Institute of Child Health, London WC1N 1EH Chris Power reader

Leah Li research fellow

Correspondence to: C Power

c.power@ich. ucl.ac.uk

BMJ 2000;320:840-1
A study investigating the relation between fetal growth and subsequent mortality of Swedish residents born in Uppsala during 1915-29 identified slow fetal growth rate rather than small size at birth as the factor associated with increased risk of ischaemic heart disease. ${ }^{1}$ The study also showed an expected association between birthweight and infant mortality. Surprisingly, birthweight was also associated with mortality in children aged 1-14 years. This association has not been previously observed, so we sought confirmation of the relation in the 1958 British birth cohort study. Because childhood death is now rare, we also examined whether birthweight had long term effects on disability.

\section{Subjects, methods, and results}

The 1958 cohort includes about 17000 people born on 3-9 March 1958 in England, Scotland, and Wales followed up to $1991 .^{2}$ Birthweight was recorded in ounces and classified into five categories (see table). Birthweight for gestational age was defined as a sex specific $Z$ score for each week of gestation. A total of 423 deaths occurring between the ages of 1 month and 38 years were notified to the NHS central register, with $19 \%$ (82) of these occurring in the first year. Disability or chronic illness and social class were ascertained at ages $7,16,23$, and 33 years. We applied logistic regression to calculate the risk of death and disability associated with birthweight and birthweight for gestational age.

For males, infant mortality was inversely associated with birthweight (odds ratio 2.13 (95\% confidence interval 1.48 to 3.07 ) per $1000 \mathrm{~g}$ reduction in birthweight). For females, infant mortality was higher for those with birthweights under $3250 \mathrm{~g}$, but not significantly, possibly because of the small numbers of deaths (table).

We found no clear associations with mortality after age 1 year. Significant inverse associations were found between birthweight and newly identified cases of disability at ages 7 (both sexes), 16 (males), and 23 (females). Risk of disability at ages 23 and 33 was raised for those with birthweights under $2500 \mathrm{~g}$, although not always significantly. Birthweight for gestational age showed similar associations with mortality and disability to those described for birthweight. Confounding by social class did not account for the relations.

\section{Comment}

Although we found the well established relation between birthweight and infant mortality, there was no evidence of a relation with childhood mortality, as seen in the Swedish sample born in 1915-29. ${ }^{1}$ Fewer deaths occurred in our 1958 cohort, and this might contribute to the discrepant findings. More plausibly, the inconsistency is due to differences in the main causes of death. Causes related to poor intrauterine growth may have been common earlier this century but have become less so as overall death rates have fallen. Unfortunately, we did not have sufficient numbers of deaths to examine separate causes. Nevertheless, our failure to detect a relation suggests that the effect of birthweight on childhood mortality has weakened. 